\title{
ER stress and autophagy are involved in the apoptosis induced by cisplatin in human lung cancer cells
}

\author{
SHAOMIN SHI ${ }^{1,2}$, PING TAN $^{2}$, BINGDI YAN $^{1}$, RONG GAO $^{1}$, JIANJUN ZHAO $^{2}$, \\ $\mathrm{JING}_{\mathrm{WANG}}{ }^{2}, \mathrm{JIA} \mathrm{GUO}^{2}, \mathrm{NING} \mathrm{LI}^{2}$ and ZHONGSEN MA ${ }^{1}$ \\ ${ }^{1}$ Department of Respiratory Medicine, The Second Hospital of Jilin University, Changchun, Jilin 130041; \\ ${ }^{2}$ Department of Respiratory Medicine, China-Japan Union Hospital of Jilin University, Changchun, Jilin 130031, P.R. China
}

Received November 25, 2015; Accepted January 12, 2016

DOI: $10.3892 /$ or.2016.4680

\begin{abstract}
Cisplatin [cis-diamminedichloroplatinum II (CDDP)] is one of the most classical and effective chemotherapeutic drugs for the treatment of cancers including lung cancer. However, the presence of cisplatin resistance in cancer lowers its curative effect and limits its usage in the clinic. The aim of the present study was to investigate the underlying mechanisms of cisplatin resistance in lung cancer involving endoplasmic reticulum (ER) stress and autophagy. In the present study, we detected the effect of cisplatin on cell viability, ER stress and autophagy in lung cancer cell lines A549 and H460. We also tested the effects of ER stress and autophagy on apoptosis induced by cisplatin. The results showed that cisplatin induced apoptosis, ER stress and autophagy in lung cancer cell lines. In addition, the inhibition of ER stress by 4-phenylbutyric acid (4-PBA) or tauroursodeoxycholic acid sodium (TUDC) enhanced cisplatin-induced apoptosis in the human lung cancer cells. Meanwhile, combination treatment with the autophagic inhibitor 3-methyladenine (3-MA) or chloroquine (CQ) further increased the apoptosis induced by cisplatin in the human lung cancer cells. The present study provides a novel treatment strategy - cisplatin in combination with an autophagic inhibitor or an ER stress inhibitor leads to increased apoptosis in human lung cancer cells.
\end{abstract}

\section{Introduction}

Cisplatin is widely considered as one of the most effective chemotherapeutic drugs for the treatment of cancers including lung cancer (1). However, cisplatin resistance of cancer cells remains an obstacle to successful chemotherapy (2). The mechanisms of cisplatin cytotoxicity include DNA damage and inhibition of DNA synthesis in cancer cells, which subse-

Correspondence to: Dr Zhongshen Ma, Department of Respiratory Medicine, The Second Hospital of Jilin University, Changchun, Jilin 130041, P.R. China

E-mail: 13331770808@163.com

Key words: apoptosis, autophagy, ER stress, human lung cancer quently prevent or enhance cancer cell death through activation of various signaling pathways including endoplasmic reticulum (ER) stress and autophagy (3).

Recent studies have shown that ER stress has a dual role, either promoting cell survival or triggering cell death depending on an imbalance between ER protein folding load and capacity $(4,5)$. ER is responsible for two key functions in eukaryotic cells, namely protein processing and intracellular calcium storage. ER stress is triggered under various physiological and pathological conditions, such as exposure to chemotherapeutic agents and accumulation of misfolded proteins $(6,7)$. However, accumulation of misfolded proteins in ER lumen resulting in ER stress causes the unfolded protein response (UPR) to initiate the expression of chaperones and proteins, such as glucose-regulated proteins [e.g. $78 \mathrm{kDa}$ glucose-regulated protein (GRP78)], calreticulin, calnexin and several folding enzymes [e.g. the thioredoxin-like protein disulfide isomerase (PDI)] (8). Moderate ER stress promotes cancer cell survival and enhances chemotherapeutic resistance, however, severe ER stress leads to cancer cell apoptosis (9). Moreover, to alleviate ER stress, the UPR signaling may activate autophagy to clear the accumulated misfolded proteins from the ER lumen (10).

Autophagy plays an important role in cell metabolism, and it can degrade intracellular macromolecules and damaged organelles to maintain cell homeostasis (11). Besides its cytoprotective role in regulating protein homeostasis, autophagy is associated with cell apoptosis as a form of programmed cell death (12). Recent research shows that cisplatin triggers autophagic cell death by damaging DNA replication (13), but chemotherapeutic drugs, including cisplatin, could induce autophagy to promote drug resistance in cancers (14). The effect of autophagy on apoptosis induced by cisplatin in lung cancer cells has not been fully understood.

In the present study, we found that cisplatin induced apoptosis, autophagy and ER stress in lung cancer cell lines. Interfering ER stress by exposure to 4-phenylbutyric acid (4-PBA) or tauroursodeoxycholic acid sodium (TUDC) enhanced the cytotoxicity induced by cisplatin in human lung cancer cells. Similarly, the autophagic inhibitor 3-methyladenine (3-MA) or chloroquine (CQ) promoted cisplatin-induced apoptosis in human lung cancer cells via inhibition of autophagy. Therefore, our data suggest that ER stress and 
autophagy may play a protective role in the apoptosis induced by cisplatin in human lung cancer cells.

\section{Materials and methods}

Cell lines and cell culture. The human lung cancer cell lines A549 and $\mathrm{H} 460$ were cultured at $37^{\circ} \mathrm{C}$ in a $5 \% \mathrm{CO}_{2}$ and $95 \%$ air atmosphere, in Roswell Park Memorial Institute (RPMI)-1640 medium (Gibco, Carlsbad, CA, USA), supplemented with 10\% fetal bovine serum (Invitrogen, Carlsbad, CA, USA), $100 \mathrm{U} / \mathrm{ml}$ penicillin and $100 \mathrm{U} / \mathrm{ml}$ streptomycin.

Cell viability assays. Cell growth inhibition (GI) was assessed by the MTT assay. Cells were seeded at densities of 8,000/well in 96-well plates. After $24 \mathrm{~h}$, various concentrations of cisplatin [cis-diamminedichloroplatinum II (CDDP)] were added to the wells and incubated for $24 \mathrm{~h}$. Each treatment was repeated in triplicate wells. Following $24 \mathrm{~h}$ of cisplatin treatment, each well received $20 \mu 1$ MTT ( $5 \mathrm{mg} / \mathrm{ml}$; Sigma-Aldrich, St. Louis, MO, USA) and was kept for $4 \mathrm{~h}$ in the dark. Then, $150 \mu \mathrm{l}$ dimethyl sulphoxide was added to dissolve the formazan crystals. Absorbance was measured with an enzyme-linked immunosorbent assay reader (Infinite M200; Tecan Group Ltd., Männedorf, Switzerland) at a wavelength of $570 \mathrm{~nm}$.

Lactate dehydrogenase ( $L D H)$ assays. The level of $\mathrm{LDH}$, as an indicator of cell injury, was measured in human lung cancer A549 and H460 cells treated with cisplatin using a standard method. The activity of the enzyme was measured with a cytotoxicity detection kit (LDH; Roche). All experiments were performed at least in triplicate.

Mitochondrial transmembrane potential (MMP) assay. The disruption of MMP was measured using fluorochrome dye 1,1',3,3'-tetraethylbenzimidazolylcarbocyanine iodide (JC-1) staining according to previously reported procedures (15). A549 and H460 cells were treated with or without different concentrations of cisplatin for $24 \mathrm{~h}$. Then, the cells were harvested and stained with $20 \mu \mathrm{M} \mathrm{JC}-1$ for $30 \mathrm{~min}$ at $37^{\circ} \mathrm{C}$ under dark conditions. Next, the cells were washed and resuspended with cold phosphate-buffered saline (PBS) for fluorescence. In addition, the wavelength was measured using a fluorescence plate reader with an excitation wavelength at $590 \mathrm{~nm}$ and an emission wavelength at $540 \mathrm{~nm}$. According to the ratio of fluorescence intensities at 590 and $540 \mathrm{~nm}$, the loss of mitochondrial membrane potential (MMP) was assessed.

Western blotting. After treatment with cisplatin alone or in combination, the cells were washed twice with cold PBS and then $120 \mu \mathrm{l}$ of RIPA buffer was added. Cell lysates were sonicated for $5 \mathrm{sec}$ on ice and then static at $4^{\circ} \mathrm{C}$ for $45 \mathrm{~min}$. After centrifuging at 3,000 $\mathrm{x}$ g for $15 \mathrm{~min}$, protein concentrations were measured using the BCA protein assay (Pierce, Rockford, IL, USA). Samples of extracted proteins were boiled for $10 \mathrm{~min}$ at $100^{\circ} \mathrm{C}$, and equivalent amounts of proteins (30-90 $\left.\mu \mathrm{g}\right)$ were separated by electrophoresis using Criterion TGX Precast 12\% gels and transferred onto Trans-Blot Turbo Midi PVDF Transfer Packs (Whatman, Maidstone, UK). The membranes were blocked with $5 \%(\mathrm{w} / \mathrm{v})$ skimmed milk for $1 \mathrm{~h}$ at room temperature, and then proteins were detected by specific primary antibodies (Santa Cruz Biotechnology, Santa Cruz, CA, USA): activated caspase-3, PARP, GRP78, cytochrome $c$, PERK and ERE1 overnight at $4^{\circ} \mathrm{C}$, followed by incubation with horseradish peroxidase-conjugated secondary antibodies $(1: 2,000$; Sigma) for $2 \mathrm{~h}$ at room temperature. The levels of proteins were quantified using Quantity One software (BioRad, Hercules, CA, USA).

Flow cytometric analysis. After the A549 and H460 cells were treated and incubated with the drugs, the cells were digested by $0.25 \%$ trypsin, and then cell death was determined using propidium iodide (PI; $1 \mu \mathrm{g} / \mathrm{ml}$ ) and Annexin V-FITC $\left(1 \mu \mathrm{g} / \mathrm{ml}\right.$; Invitrogen). After a $15-\mathrm{min}$ incubation at $37^{\circ} \mathrm{C}$, the samples were detected using a FACScan flow cytometer (BD Biosystems, San Jose, CA, USA). All experiments in the present study were performed in triplicate.

Statistical analysis. All data were analyzed using the t-test. $\mathrm{P}<0.05$ was considered to indicate a statistically significant difference. Data in the present study are representative of three independent experiments performed in triplicate.

\section{Results}

Cisplatin inhibits the cell proliferation of human lung cancer A549 and H460 cells. Human lung cancer A549 and H460 cells were treated with varying doses of cisplatin for $24 \mathrm{~h}$, and then the cell viability rate was examined by MTT assay. According to the results, we found that cisplatin inhibited A549 and H460 cell proliferation in a dose-dependent manner (Fig. 1A and B). Meanwhile, the cell death of the treated A549 and H460 cells was assessed via measuring the leakage of LDH. The result showed that the level of LDH was obviously increased in the A549 and H460 cells treated with cisplatin, and cisplatin significantly increased the release of LDH in a dose-dependent manner (Fig. 1C and D). These results indicate that cisplatin inhibited the cell growth of human lung cancer A549 and H460 cells.

Cisplatin induces human lung cancer A549 and H460 cell death by mitochondrial-pathway apoptosis. After treatment with cisplatin, we next aimed to ascertain the mechanism involved in the cisplatin-induced apoptosis of human lung cancer A549 and H460 cells. Compared with the control, the apoptotic rate was obviously increased in the A549 and H460 cells following treatment with 10,20 and $40 \mu \mathrm{M}$ cisplatin which was detected by flow cytometric analysis (Fig. 2A and B). Meanwhile, we assessed the apoptotic effects of cisplatin on A549 and H460 cells, through examination of the activation of caspase- 3 and PARP using western blotting. The levels of activation of caspase-3 and PARP were significantly increased in the A549 and $\mathrm{H} 460$ cells following treatment with cisplatin (Fig. 2C-F). To assess the exact mechanism of cisplatin-induced apoptosis, we detected the intrinsic mitochondrial-mediated apoptotic pathway in the A549 and H460 cells. As shown in Fig. 3A and B, cisplatin obviously reduced the mitochondrial potential in the A549 and $\mathrm{H} 460$ cells treated with cisplatin. In addition, cisplatin upregulated the level of cytosolic cytochrome $c$ in the A549 and H460 cells (Fig. 3C-F). These results indicated that cisplatin signifi- 
A

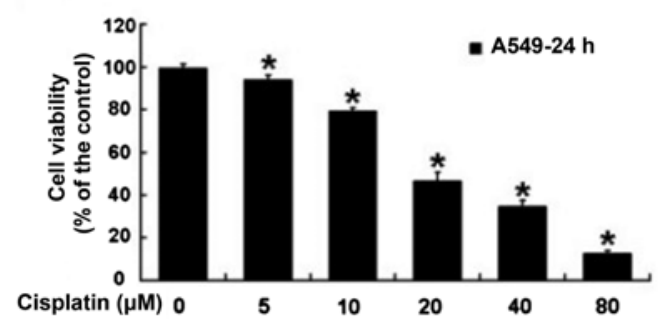

C

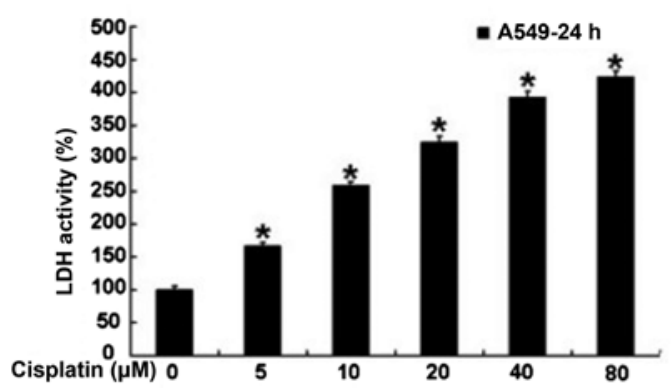

B

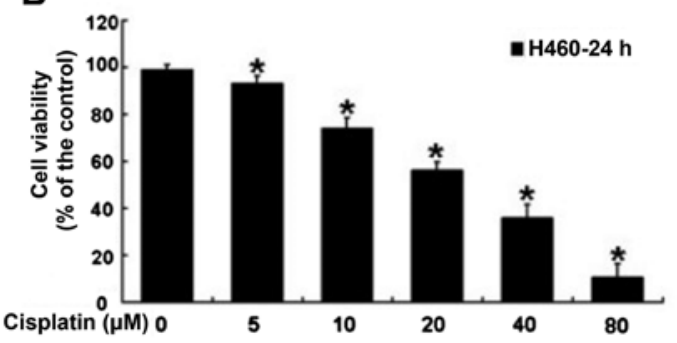

D

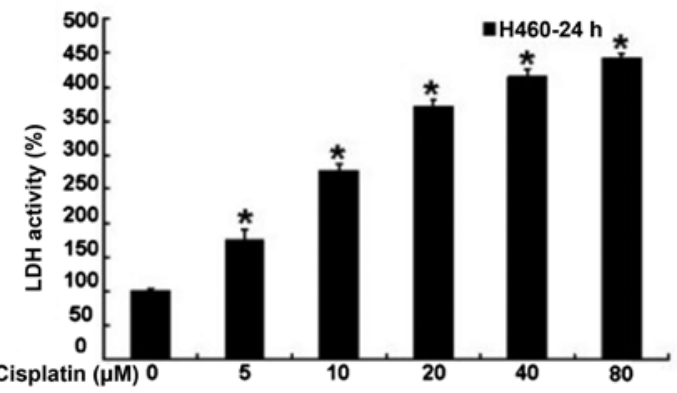

Figure 1. Cisplatin inhibits A549 and H460 cell proliferation. (A and B) A549 and H460 cells were treated with 5, 10, 20, 40 and $80 \mu \mathrm{M}$ cisplatin for 24 h. Cell viability was detected by MTT assay. Data are presented as mean \pm SD, $n=6$. ${ }^{*} \mathrm{P}<0.05$ vs. the control group. (C and D) A549 and H460 cells were treated in the same manner as in A for $24 \mathrm{~h}$. LDH activity was detected. Data are presented as mean $\pm \mathrm{SD}, \mathrm{n}=6$. ${ }^{\mathrm{P}} \mathrm{P}<0.05$ vs. the control group.

A

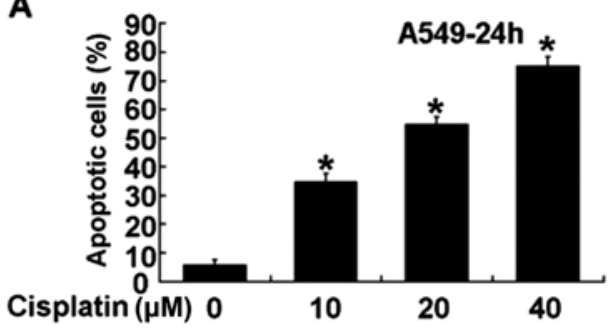

C

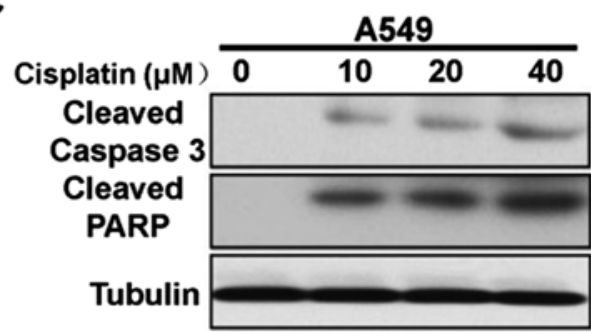

E

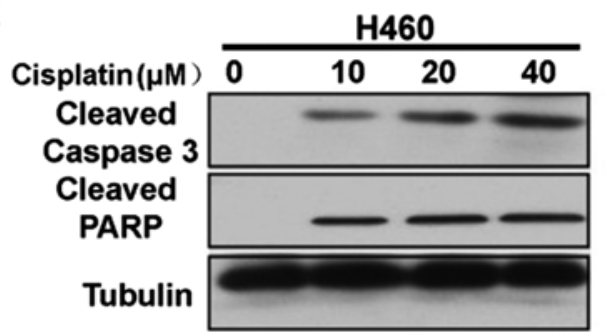

B

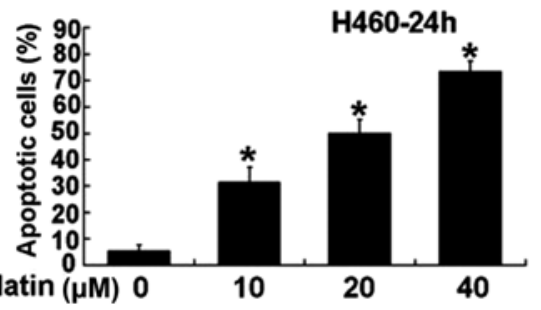

D

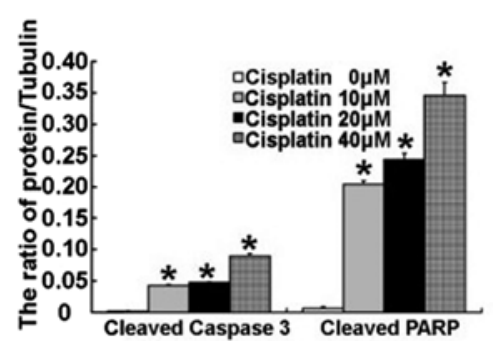

$\mathbf{F}$

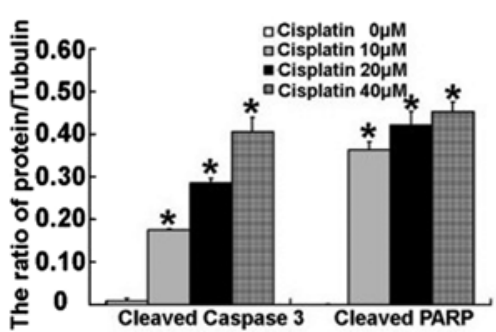

Figure 2. Cisplatin induces A549 and $\mathrm{H} 460$ cell apoptosis via the mitochondrial-associated pathway (A and B) After treatment with 10,20 and $40 \mu \mathrm{M}$ cisplatin, cell apoptosis was detected using flow cytometry in the A549 and H460 cells for $24 \mathrm{~h}$. (C and E) The expression of cleaved caspase-3 and cleaved PARP was detected by western blotting in the A549 and H460 cells treated with different concentrations of cisplatin for $24 \mathrm{~h}$. (D and F) Quantitation of cleaved caspase-3 and cleaved PARP protein levels. Data are presented as mean $\pm \mathrm{SD}, \mathrm{n}=3,{ }^{*} \mathrm{P}<0.05$ vs. the control group.

cantly induced the apoptosis of A549 and $\mathrm{H} 460$ cells through the intrinsic mitochondrial apoptotic pathway.
Cisplatin induces ER stress in human lung cancer A549 and $H 460$ cells. To further assess whether ER stress is associated 
A

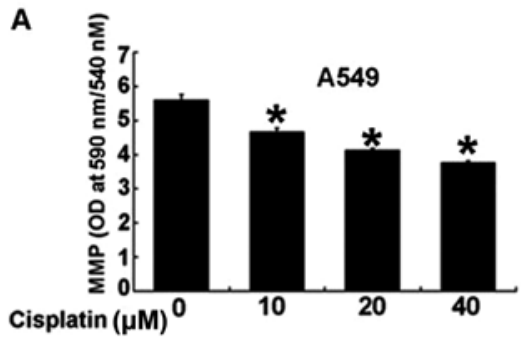

C

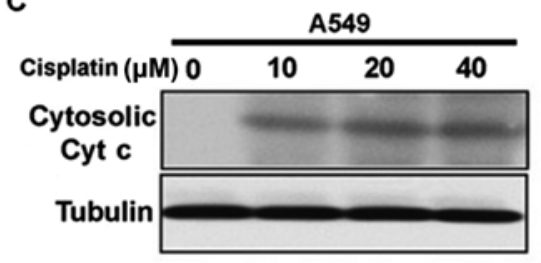

E

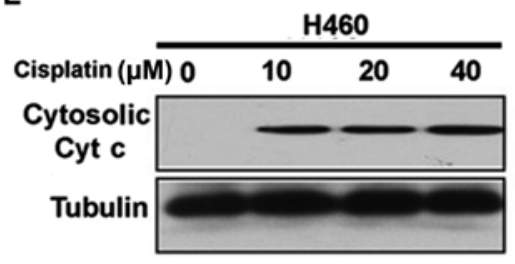

B

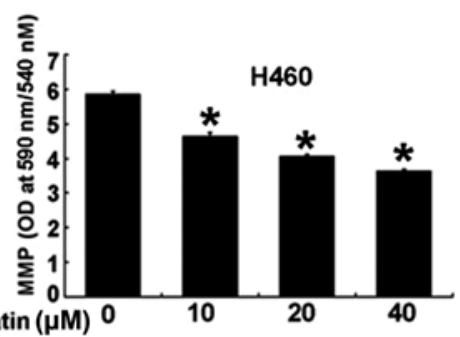

D

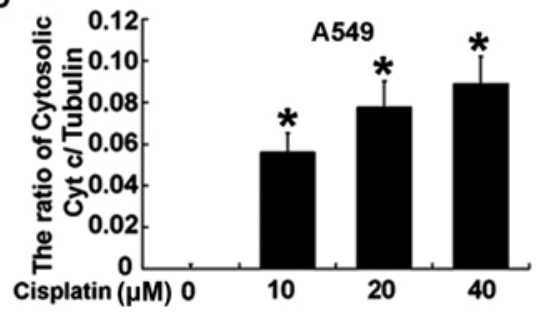

$F$

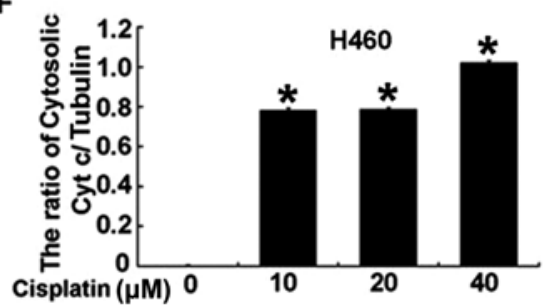

Figure 3. Cisplatin induces A549 and H460 cell apoptosis via the mitochondrial-associated pathway. (A and B) Mitochondrial transmembrane potential (MMP) was detected by JC-1 staining using a fluorescence plate reader in the A549 and H460 cells treated with 10,20 and $40 \mu \mathrm{M}$ cisplatin for 24 h. (C and E) The expression of cytosolic cytochrome $c$ (cyt $c$ ) was detected by western blotting in the A549 and H460 cells treated with different concentrations of cisplatin for $24 \mathrm{~h}$. (D and F) Quantitation of the cytosolic cyt $c$ protein levels. Data are presented as mean $\pm \mathrm{SD}, \mathrm{n}=3$, ${ }^{*} \mathrm{P}<0.05$ vs. the control group.

with the mechanism of cisplatin-induced apoptosis in human lung cancer A549 and H460 cells, we examined expression of ER stress proteins in the cells following treatment with cisplatin. After A549 and H460 cells were treated with 10, 20 and $40 \mu \mathrm{M}$ cisplatin for $24 \mathrm{~h}$, we detected the expression of Grp78, an ER stress marker. Using western blotting, we found that the expression of Grp78 was increased in the A549 and H460 cells treated with cisplatin (Fig. 4A-D). When ER stress is induced, it triggers the UPR to respond to environmental factors. Thus UPR regulates the expression of the ER stress sensor protein PKR-like ER kinase (PERK), and the inositol-requiring enzyme 1 (IRE-1). As shown in Fig. 4E-H, expression of ER stress-associated proteins PERE/IRE1 were increased in the A549 and H460 cells following treatment with cisplatin. These results demonstrated that ER stress was induced in the A549 and $\mathrm{H} 460$ cells following treatment with cisplatin, and that this was regulated by the PERK and IRE1 pathway.

ER stress is involved in cisplatin-induced apoptosis in human lung cancer A549 and H460 cells. We next wanted to examine the relevance of ER stress in cisplatin-induced apoptosis in human lung cancer A549 and H460 cells. We used ER stress inhibitors 4-PBA and TUDC to alleviate ER stress in the A549 and H460 cells treated with cisplatin. Using MTT assay, we found that inhibition of ER stress effectively decreased the cell viability of the cisplatin-treated A549 and H460 cells at $24 \mathrm{~h}$ (Fig. 5A and B). Consistent with the inhibition of cell viability, inhibition of ER stress significantly enhanced the cell apoptosis of the cisplatin-treated A549 and
H460 cells at $24 \mathrm{~h}$ (Fig. 5C and D). Then, we examined the expression of apoptosis-associated proteins, cleaved caspase-3 and cytosolic cytochrome $c$. According to the results, activation of caspase-3 was obviously upregulated, and the level of cytosolic cytochrome $c$ was also significantly increased (Figs. 5E and F and 6A-F). These results demonstrated that cisplatin can induce ER stress, and inhibition of ER stress increased the apoptosis in the A549 and H460 cells treated with cisplatin.

Cisplatin induces the activation of autophagy in human lung cancer $A 549$ and $H 460$ cells. In addition to regulating ER stress, we also found activation of autophagy in the A549 and H460 cells treated with cisplatin. Thus, we examined the expression of the protein Beclin 1, which is required for autophagy. After A549 and H460 cells were treated with 10, 20 and $40 \mu \mathrm{M}$ cisplatin for $24 \mathrm{~h}$, we found that expression of Beclin 1 was significantly upregulated in the A549 and H460 cells (Fig. 7A-D). Next, LC3, a molecular marker of autophagy, was assessed. We found that the ratio of LC3 II/LC3 I was high in the A549 and H460 cells treated by cisplatin (Fig. 7A-D). These results demonstrated that autophagy was induced by cisplatin in the A549 and H460 cells.

Inhibition of autophagy enhances cisplatin-induced cytotoxicity in human lung cancer A549 and H460 cells. The previous results demonstrated that cisplatin induced activation of autophagy and apoptosis. However, the mechanisms of autophagy and apoptosis are still unclear, but important 
A

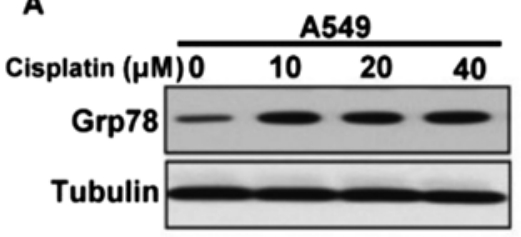

B

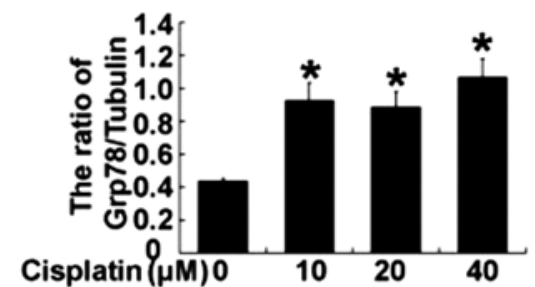

E

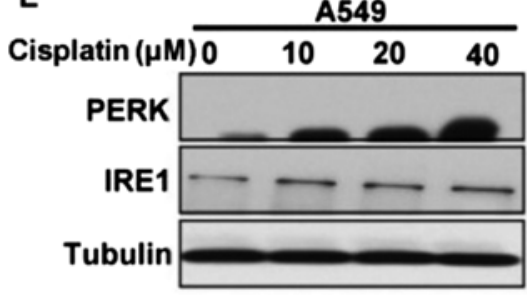

$\mathbf{F}$

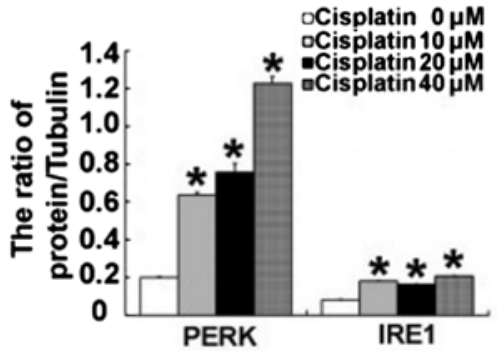

C

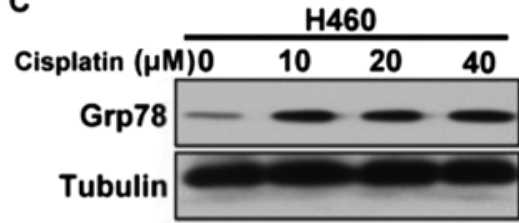

D

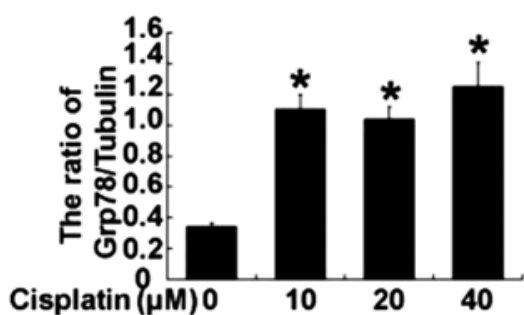

G

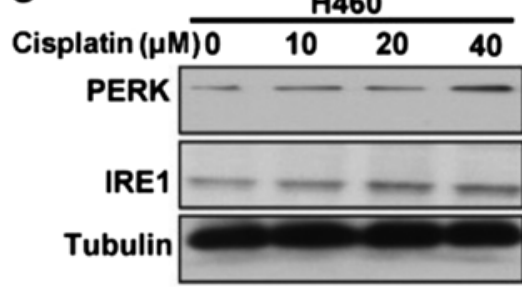

H

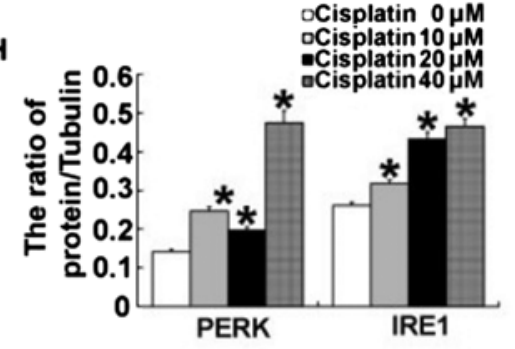

Figure 4. Cisplatin-induced apoptosis is associated with ER stress-associated pathway. A549 and H460 cells were treated with 10, 20 and $40 \mu \mathrm{M}$ cisplatin for $24 \mathrm{~h}$. (A and C) The expression of GRP78, ER stress protein, was detected by western blotting. (B and D) The level of protein GRP78 was quantified. Data are presented as mean $\pm \mathrm{SD}, \mathrm{n}=3,{ }^{*} \mathrm{P}<0.05$ vs. control group. (E and G) The expression levels of ER stress-associated apoptosis proteins PERK and IRE1 were analyzed by western blotting. ( $\mathrm{F}$ and $\mathrm{H}$ ) The levels of PERK and IRE1 protein were quantified. Data are presented as mean $\pm \mathrm{SD}$, $\mathrm{n}=3$, $\mathrm{P}<0.05$ vs. control group.

in cancer treatment. Next, we explored whether there was a relationship between autophagy and apoptosis in the A549 and H460 cells treated with cisplatin. To clarify the action of autophagy in the A549 and H460 cells treated with cisplatin, we used two different autophagy inhibitors, 3-MA (which inhibits the initiation of autophagy) and CQ (which blocks the fusion of autophagosomes and lysosomes). As shown in Fig. 8A-D, we found that combination of 3-MA or CQ and cisplatin further decreased the cell viability and induced the cell apoptosis of A549 and H460 cells. When we inhibited autophagy by 3-MA and CQ, we found that the level of apoptosis-associated proteins, activated caspase- 3 and cytosolic cytochrome $c$, were obviously increased in the A549 and H460 cells treated with cisplatin (Figs. 8E and $\mathrm{F}$ and $9 \mathrm{~A}-\mathrm{F}$ ). These results suggest that inhibition of autophagy enhanced cisplatin-induced cytotoxicity and apoptosis in the human lung cancer cells.

\section{Discussion}

Lung cancer is one of the most common malignancies, and cisplatin is often used as a first-line chemotherapeutic agent in cancers including lung cancer. However, lung cancer cells become resistant to cisplatin and it is an obstacle in clinical treatment (16-18). Numerous studies have shown that cisplatin induces the mitochondrial apoptosis pathway by disturbing mitochondrial potential, thus resulting in a caspase-dependent intrinsic pathway (19-21). Recent studies show that cisplatin induces ER stress as well, and sustained and severe ER stress results in activation of caspase-mediated apoptosis in cancers $(22,23)$. However, the exact mechanisms of cisplatin-induced cell death in human lung cancer cells are not fully understood. Consistently in the present study, we found that cisplatin triggered the apoptosis of human lung cancer A549 and H460 cells through classic caspase-mediated apoptosis. Cisplatin also altered mitochondrial potential, thus leading to activation of mitochondrial apoptotic pathways through classic cytochrome $c$ and caspase-3.

ER is a key organelle with protein processing, intracellular calcium storage, as well as crucial biosynthetic and signaling regulation functions in eukaryotic cells (24). When destroying an imbalance between ER protein folding load and capacity in different physiological and pathological conditions, such 
A

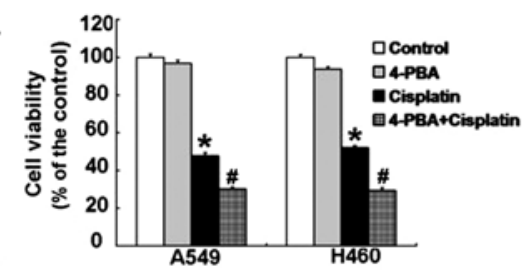

C

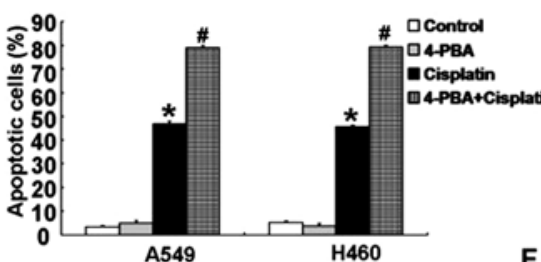

E

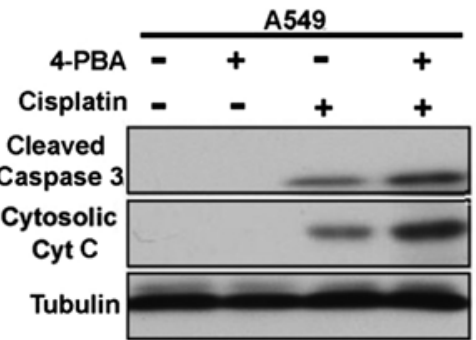

B
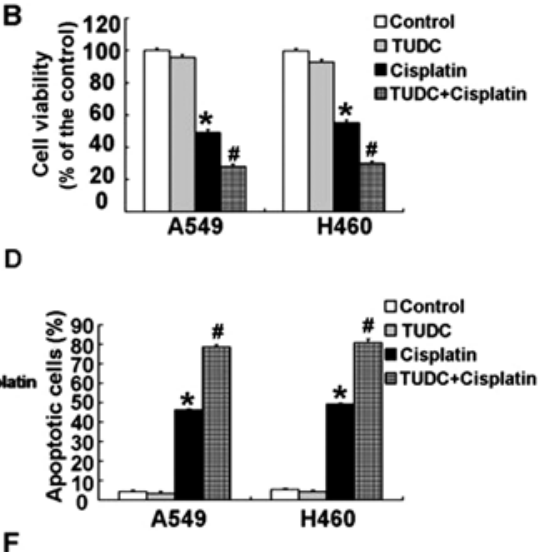

$\mathbf{F}$

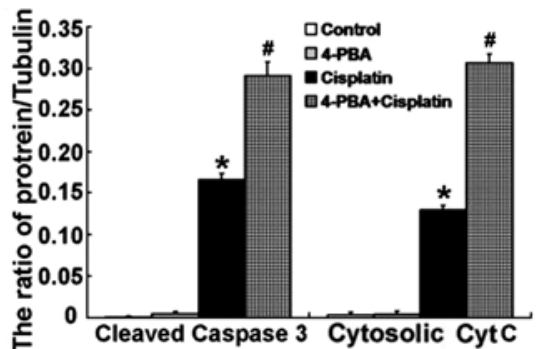

Figure 5. Inhibition of ER stress enhances the cytotoxicity of cisplatin in A549 and H460 cells. A549 and H460 cells were treated with a combination of $20 \mu \mathrm{M}$ cisplatin and $500 \mu \mathrm{M} 4$-PBA or $500 \mu \mathrm{M}$ TUDC for $24 \mathrm{~h}$. (A and B) Cell viability was detected by MTT assay in the A549 and H460 cells treated with a combination of $20 \mu \mathrm{M}$ cisplatin and 4-PBA or TUDC for $24 \mathrm{~h}$. (C and D) Being subjected to the same treatment, cell apoptosis was detected using flow cytometry. (E) Western blot analysis of the expression of cleaved caspase-3 and cytosolic cytochrome $c$ (cyt $c$ ) proteins in the A549 cells treated with cisplatin and/or 4-PBA for $24 \mathrm{~h}$. (F) Quantitative analysis of cleaved caspase-3 and cytosolic cyt $c$ protein levels. Data are presented as mean $\pm \mathrm{SD}$, $\mathrm{n}=3$, ${ }^{\mathrm{P}}<0.05$ vs. the control group, and ${ }^{\#} \mathrm{P}<0.05$ vs. the cisplatin group.

A

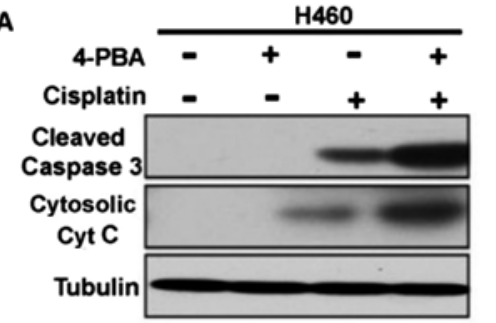

C
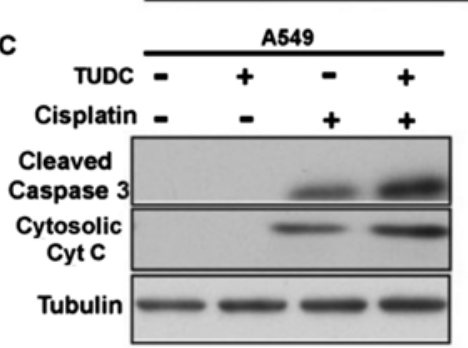

E

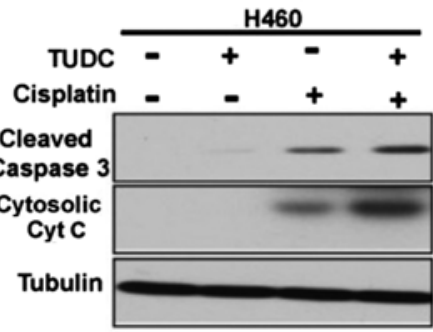

B c

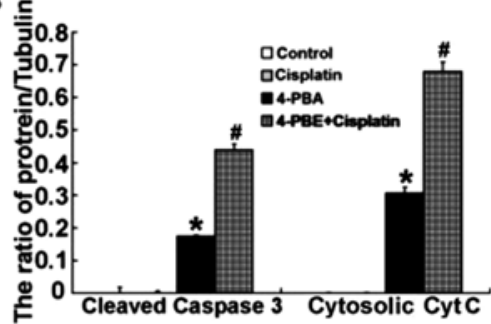

D

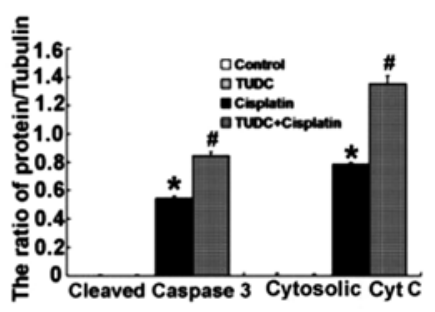

$\mathbf{F}$

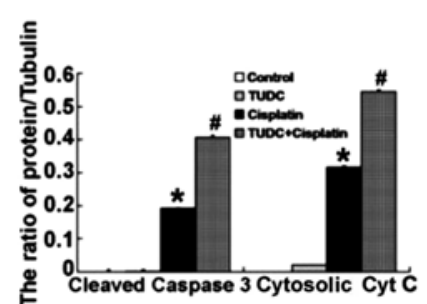

Figure 6. Inhibition of ER stress enhances the cytotoxicity of cisplatin in A549 and H460 cells. (A) Western blot analysis of the expression of cleaved caspase-3 and cytosolic cytochrome $c$ (cyt $c$ ) proteins in H460 cells treated with cisplatin and/or 4-PBA for $24 \mathrm{~h}$. (B) Quantitative analysis of cleaved caspase-3 and cytosolic cyt $c$ protein levels. Data are presented as mean $\pm \mathrm{SD}, \mathrm{n}=3,{ }^{*} \mathrm{P}<0.05$ vs. control group and ${ }^{\#} \mathrm{P}<0.05$ vs. cisplatin group. (C and $\mathrm{E}$ ) Western blot analysis of the expression of cleaved caspase-3 and cytosolic cyt $c$ proteins in A549 and H460 cells treated with cisplatin and/or TUDC for 24 h. (D and F) The levels of cleaved caspase- 3 and cytosolic cyt $c$ protein were quantified. Data are presented as mean $\pm \mathrm{SD}, \mathrm{n}=3,{ }^{*} \mathrm{P}<0.05$ vs. control group and ${ }^{\#} \mathrm{P}<0.05$ vs. $20 \mu \mathrm{M}$ cisplatin group. 
A

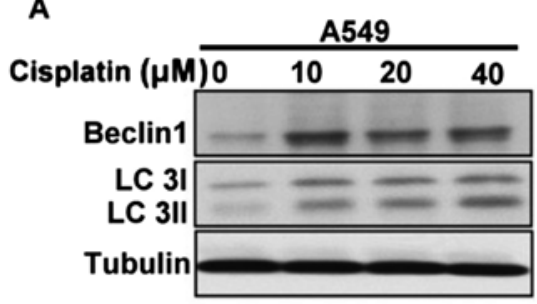

C

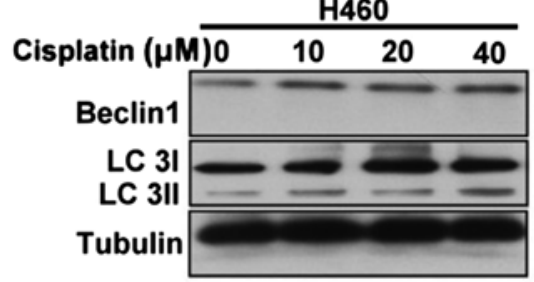

B

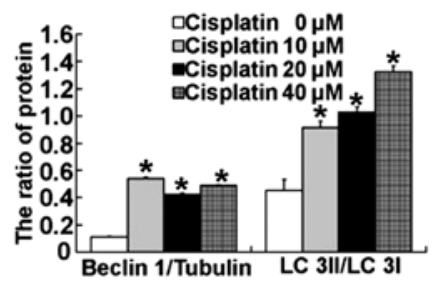

D

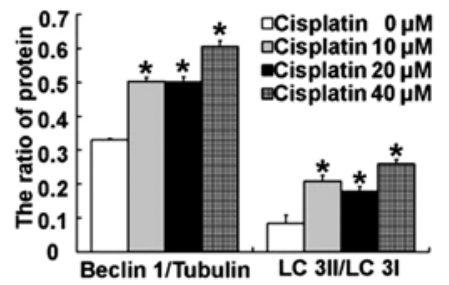

Figure 7. Cisplatin induces autophagy in A549 and $\mathrm{H} 460$ cells treated with 10, 20 and $40 \mu \mathrm{M}$ cisplatin for $24 \mathrm{~h}$. (A and C) Western blot analysis of the expression of Beclin 1 and LC 3II/LC 3I proteins in A549 and H460 cells. (B and D) Quantitative analysis of Beclin 1 and LC 3II/LC 3I protein levels. Data are presented as mean $\pm \mathrm{SD}, \mathrm{n}=3,{ }^{*} \mathrm{P}<0.05$ vs. the control group.

A

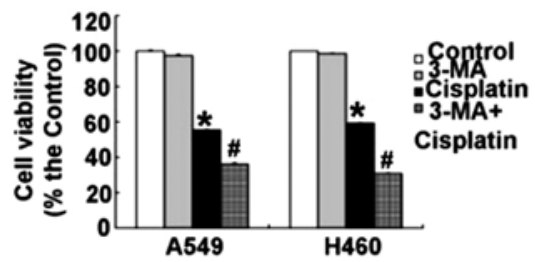

C

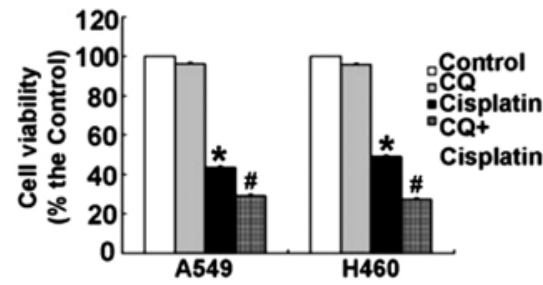

E

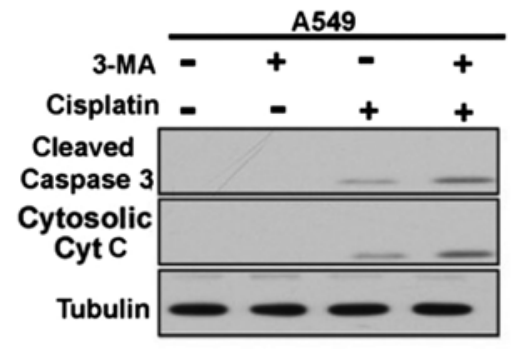

B

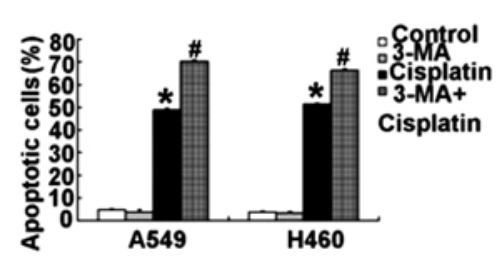

D

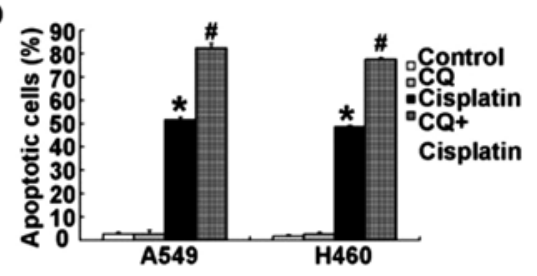

$\mathbf{F}$

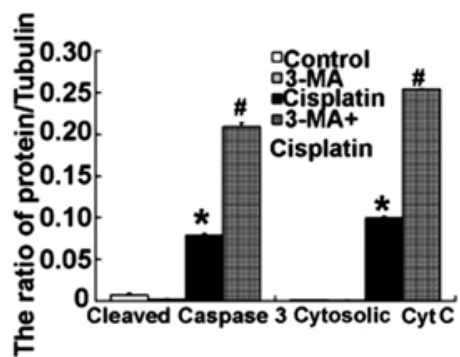

Figure 8. Inhibition of autophagy by 3-MA and CQ enhances cisplatin-induced cell death. A549 and H460 cells were treated with $20 \mu \mathrm{M}$ cisplatin and $5 \mathrm{mM}$ 3-MA or $50 \mu \mathrm{M} \mathrm{CQ}$ for $24 \mathrm{~h}$. (A and C) Cell viability was detected by MTT assay in the A549 and H460 cells treated with a combination of $20 \mu \mathrm{M}$ cisplatin and 3-MA/CQ for $24 \mathrm{~h}$. (B and D) Subjected to the same treatment, cell apoptosis was detected using flow cytometry. (E) Western blot analysis of the expression of cleaved caspase-3 and cytosolic cytochrome $c$ (cyt $c$ ) protein in A549 cells treated with a combination of $20 \mu \mathrm{M}$ cisplatin and $5 \mathrm{mM} 3-\mathrm{MA}$ for $24 \mathrm{~h}$. (F) Quantitative analysis of cleaved caspase-3 and cytosolic cyt $c$ protein levels. Data are presented as mean $\pm \mathrm{SD}, \mathrm{n}=3$, ${ }^{*} \mathrm{P}<0.05$ vs. the control group and ${ }^{\#} \mathrm{P}<0.05$ vs. the $20 \mu \mathrm{M}$ cisplatin group.

as cisplatin, this results in accumulated unfolded proteins of ER lumen, known as 'ER stress'. ER stress can initiate cell UPR, which regulates the expression of ER molecular chaperone GRP78/Bip, the ER stress sensor protein PERK, the inositol-requiring enzyme 1 (IRE-1). Mild to moderate ER stress promotes cell survival through UPR to alleviate ER stress (25). In addition, it has been proven that human lung cancer cells can acquire cisplatin-resistance via ER stress (26).
However sustained and severe ER stress or inhibition of ER stress leads to cell death in some cancers including lung cancer (27). However, the exact function of cisplatin-induced ER stress is not fully defined. In the present study, we demonstrated that cisplatin induced ER stress through the expression of GRP78, IRE1 and PERK. However, we could not detect the expression of ER stress associated protein CHOP. It may be due to the low level of the ER stress induced by cisplatin 
A

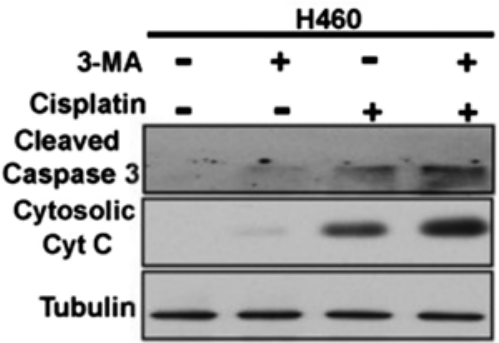

C

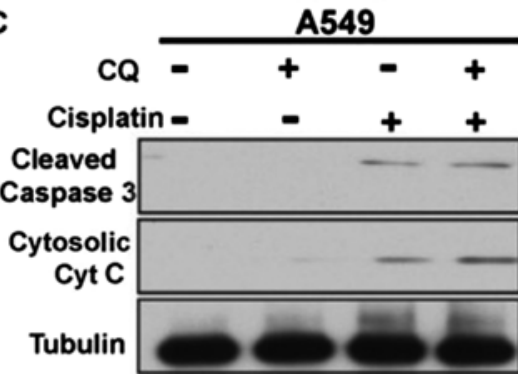

$\mathbf{E}$

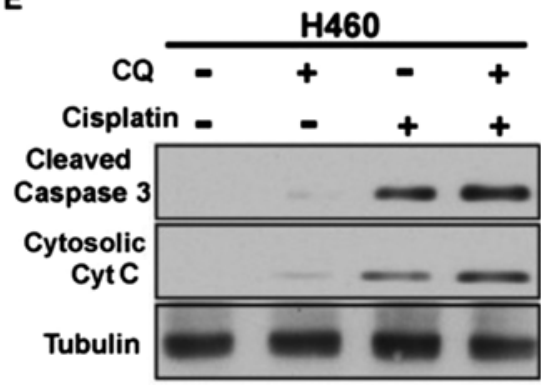

B

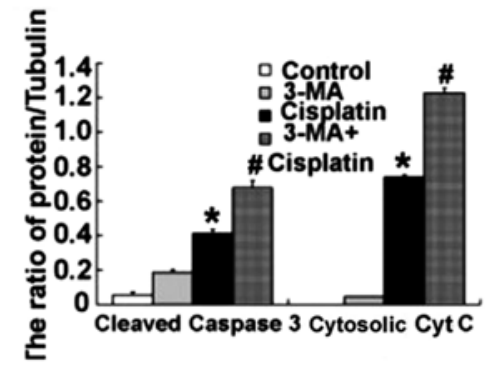

D

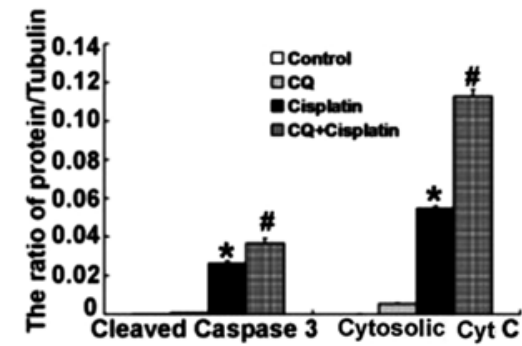

$\mathbf{F}$

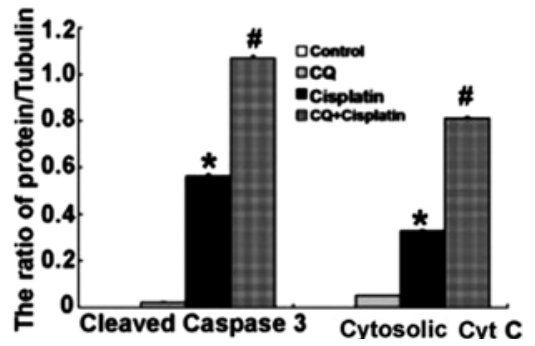

Figure 9. Inhibition of autophagy by 3-MA and CQ enhances cisplatin-induced cell death. (A) Western blot analysis of the expression of cleaved caspase-3 and cytosolic cytochrome $c$ (cyt $c$ ) protein in the $\mathrm{H} 460$ cells treated with a combination of $20 \mu \mathrm{M}$ cisplatin and $5 \mathrm{mM} 3-\mathrm{MA}$ for $24 \mathrm{~h}$. (B) Quantitative analysis of cleaved caspase- 3 and cytosolic cyt $c$ protein levels. Data are presented as mean $\pm \mathrm{SD}, \mathrm{n}=3,{ }^{*} \mathrm{P}<0.05$ vs. the control group and ${ }^{\#} \mathrm{P}<0.05 \mathrm{vs}$. the $20 \mu \mathrm{M}$ cisplatin group. (C and E) Western blot analysis of the expression of cleaved caspase-3 and cytosolic cyt $c$ protein in the A549 and H460 cells treated with a combination of $20 \mu \mathrm{M}$ cisplatin and $50 \mu \mathrm{M} \mathrm{CQ}$ for $24 \mathrm{~h}$. (D and F) Quantitative analysis of cleaved caspase-3 and cytosolic cyt $c$ protein levels. Data are presented as mean $\pm \mathrm{SD}, \mathrm{n}=3,{ }^{*} \mathrm{P}<0.05$ vs. the control group and ${ }^{\#} \mathrm{P}<0.05$ vs. the $20 \mu \mathrm{M}$ cisplatin group.

which could not induce ER stress-associated apoptosis. When we blocked ER stress by ER stress inhibitors 4-PBA or TUDC, cytotoxicity and apoptosis were obviously increased in the human lung cancer A549 and H460 cells treated with cisplatin. Besides ER stress, it is reported that some antitumor agents can induced autophagy $(28,29)$. Meanwhile, some studies showed that UPR signaling may activate autophagy to alleviate ER stress through the PERK signaling pathway (30).

Autophagy is an intracellular metabolic system in eukaryotic cells, in which autophagosomes fuse with the lysosome, and degrade intracellular materials to maintain cell homeostasis (31). Various studies have shown that activation of autophagy protects cells from cisplatin-induced apoptosis, allowing cells to alleviate ER stress, consequently causing cisplatin resistance $(14,32)$. However, the role of autophagy in human lung cancer cells treated with cisplatin is still not clear. According to the expression of LC3 and Beclin 1, we demonstrated that cisplatin induced autophagy in human lung cancer A549 and H460 cells. However, it is unclear whether activation of autophagy protects cells from cisplatin-induced apoptosis or leads to cell death in human lung cancer cells. Our results showed that inhibition of autophagy by 3-MA or CQ enhanced cisplatin-induced apoptosis in human lung cancer
A549 and $\mathrm{H} 460$ cells by triggering the mitochondrial-apoptosis pathway. These data indicate that autophagy plays a protective role and is involved in cisplatin resistance in human lung cancer A549 and H460 cells.

In summary, we found that cisplatin can induce apoptosis, ER stress and autophagy, and ER stress and autophagy are involved in the mechanism of cisplatin resistance in human lung cancer cells. Inhibition of ER stress or autophagy can further increase the apoptosis induced by cisplatin. Therefore, our data suggest that cisplatin-induced ER stress and autophagy may play a protective role in apoptosis induced by cisplatin in human lung cancer cells.

\section{References}

1. Lee HY, Mohammed KA, Goldberg EP, Kaye F and Nasreen N: Cisplatin loaded albumin mesospheres for lung cancer treatment. Am J Cancer Res 5: 603-615, 2015.

2. Wu DW, Lee MC, Hsu NY, Wu TC, Wu JY, Wang YC, Cheng YW, Chen CY and Lee H: FHIT loss confers cisplatin resistance in lung cancer via the $\mathrm{AKT} / \mathrm{NF}-\kappa \mathrm{B} / \mathrm{Slug}$-mediated PUMA reduction. Oncogene 34: 2546, 2015.

3. Zhang R, Wang R, Chen Q and Chang H: Inhibition of autophagy using 3-methyladenine increases cisplatin-induced apoptosis by increasing endoplasmic reticulum stress in U251 human glioma cells. Mol Med Rep 12: 1727-1732, 2015. 
4. Jang JH, Kim YJ, Kim H, Kim SC and Cho JH: Buforin IIb induces endoplasmic reticulum stress-mediated apoptosis in HeLa cells. Peptides 69: 144-149, 2015.

5. Shen Y, Yang J, Zhao J, Xiao C, Xu C and Xiang Y: The switch from ER stress-induced apoptosis to autophagy via ROS-mediated JNK/p62 signals: A survival mechanism in methotrexate-resistant choriocarcinoma cells. Exp Cell Res 334: 207-218, 2015

6. Lin YD, Chen S, Yue P, Zou W, Benbrook DM, Liu S, Le TC, Berlin KD, Khuri FR and Sun SY: CAAT/enhancer binding protein homologous protein-dependent death receptor 5 induction is a major component of SHetA2-induced apoptosis in lung cancer cells. Cancer Res 68: 5335-5344, 2008.

7. Ron D and Hubbard SR: How IRE1 reacts to ER stress. Cell 132: 24-26, 2008

8. Dong D, Ni M, Li J, Xiong S, Ye W, Virrey JJ, Mao C, Ye R, Wang M, Pen L, et al: Critical role of the stress chaperone GRP78/BiP in tumor proliferation, survival, and tumor angiogenesis in transgene-induced mammary tumor development. Cancer Res 68: 498-505, 2008

9. Szegezdi E, Logue SE, Gorman AM and Samali A: Mediators of endoplasmic reticulum stress-induced apoptosis. EMBO Rep 7: 880-885, 2006.

10. Kouroku Y, Fujita E, Tanida I, Ueno T, Isoai A, Kumagai H, Ogawa S, Kaufman RJ, Kominami E and Momoi T: ER stress (PERK/eIF2alpha phosphorylation) mediates the polyglutamine-induced LC3 conversion, an essential step for autophagy formation. Cell Death Differ 14: 230-239, 2007.

11. Li MQ and Liu ZG: Dual role of autophagy in chronic myeloid leukemia. Zhongguo Shi Yan Xue Ye Xue Za Zhi 23: 583-586, 2015 (In Chinese).

12. Kim MO, Lee HS, Chin YW, Moon DO and Ahn JS: Gartanin induces autophagy through JNK activation which extenuates caspase-dependent apoptosis. Oncol Rep 34: 139-146, 2015.

13. He J, Yu JJ, Xu Q, Wang L, Zheng JZ, Liu LZ and Jiang BH: Downregulation of ATG14 by EGR1-MIR152 sensitizes ovarian cancer cells to cisplatin-induced apoptosis by inhibiting cyto-protective autophagy. Autophagy 11: 373-384, 2015.

14. Bao L, Jaramillo MC, Zhang Z, Zheng Y, Yao M, Zhang DD and Yi X: Induction of autophagy contributes to cisplatin resistance in human ovarian cancer cells. Mol Med Rep 11: 91-98, 2015

15. Shen H, Zeng G, Sun B, Cai X, Bi L, Tang G and Yang Y: A polysaccharide from Glycyrrhiza inflata Licorice inhibits proliferation of human oral cancer cells by inducing apoptosis via mitochondrial pathway. Tumour Biol 36: 4825-4831, 2015.

16. Köberle B, Tomicic MT, Usanova S and Kaina B: Cisplatin resistance: Preclinical findings and clinical implications. Biochim Biophys Acta 1806: 172-182, 2010.

17. Chen J, Solomides C, Parekh H, Simpkins F and Simpkins H: Cisplatin resistance in human cervical, ovarian and lung cancer cells. Cancer Chemother Pharmacol 75: 1217-1227, 2015.
18. Horibe S, Matsuda A, Tanahashi T, Inoue J, Kawauchi S, Mizuno S, Ueno M, Takahashi K, Maeda Y, Maegouchi T, et al: Cisplatin resistance in human lung cancer cells is linked with dysregulation of cell cycle associated proteins. Life Sci 124: 31-40, 2015

19. Zhu J, Yang Y and Wu J: Bcl-2 cleavages at two adjacent sites by different caspases promote cisplatin-induced apoptosis. Cell Res 17: 441-448, 2007.

20. Sharma H, Sen S and Singh N: Molecular pathways in the chemosensitization of cisplatin by quercetin in human head and neck cancer. Cancer Biol Ther 4: 949-955, 2005.

21. Wei Q, Dong G, Franklin J and Dong Z: The pathological role of Bax in cisplatin nephrotoxicity. Kidney Int 72: 53-62, 2007.

22. Liu Z, Sun Y, Ren L, Huang Y, Cai Y, Weng Q, Shen X, Li X, Liang $G$ and Wang Y: Evaluation of a curcumin analog as an anti-cancer agent inducing ER stress-mediated apoptosis in non-small cell lung cancer cells. BMC Cancer 13: 494, 2013.

23. Wu SH, Hang LW, Yang JS, Chen HY, Lin HY, Chiang JH, Lu CC, Yang JL, Lai TY, Ko YC, et al: Curcumin induces apoptosis in human non-small cell lung cancer NCI-H460 cells through ER stress and caspase cascade- and mitochondria-dependent pathways. Anticancer Res 30: 2125-2133, 2010.

24. Moir RD, Gross DA, Silver DL and Willis IM: $S C S 3$ and YFT2 link transcription of phospholipid biosynthetic genes to ER stress and the UPR. PLoS Genet 8: e1002890, 2012.

25. Hetz C: The UPR as a survival factor of cancer cells: More than folding proteins? Leuk Res 33: 880-882, 2009.

26. Lin Y, Wang Z, Liu L and Chen L: Akt is the downstream target of GRP78 in mediating cisplatin resistance in ER stress-tolerant human lung cancer cells. Lung Cancer 71: 291-297, 2011.

27. Cui WY, Liu Y, Zhu YQ, Song T and Wang QS: Propofol induces endoplasmic reticulum (ER) stress and apoptosis in lung cancer cell H460. Tumour Biol 35: 5213-5217, 2014.

28. Lan D, Wang W, Zhuang J and Zhao Z: Proteasome inhibitorinduced autophagy in PC12 cells overexpressing A53T mutant $\alpha$-synuclein. Mol Med Rep 11: 1655-1660, 2015.

29. Zang Y, Thomas SM, Chan ET, Kirk CJ, Freilino ML, DeLancey HM, Grandis JR, Li C and Johnson DE: The next generation proteasome inhibitors carfilzomib and oprozomib activate prosurvival autophagy via induction of the unfolded protein response and ATF4. Autophagy 8: 1873-1874, 2012.

30. Tallóczy Z, Jiang W, Virgin HW IV, Leib DA, Scheuner D, Kaufman RJ, Eskelinen EL and Levine B: Regulation of starvation- and virus-induced autophagy by the eIF2alpha kinase signaling pathway. Proc Natl Acad Sci USA 99: 190-195, 2002.

31. Liu M, Ma S, Liu M, Hou Y, Liang B, Su X and Liu X Synergistic killing of lung cancer cells by cisplatin and radiation via autophagy and apoptosis. Oncol Lett 7: 1903-1910, 2014.

32. Bao LJ, Jaramillo MC, Zhang ZB, Zheng YX, Yao M, Zhang DD and Yi XF: Nrf2 induces cisplatin resistance through activation of autophagy in ovarian carcinoma. Int J Clin Exp Pathol 7: 1502-1513, 2014. 